\title{
Application of parabolic equation methods to in-ice radio wave propagation for ultra high energy neutrino detection experiments
}

\section{Cade Sbrocco $^{a, *}$ and Steven Prohira ${ }^{a}$ on behalf of the Radar Echo Telescope Collaboration}

(a complete list of authors can be found at the end of the proceedings)

${ }^{a}$ Department of Physics, Center for Cosmology and AstroParticle Physics (CCAPP), The Ohio State University, Columbus OH, USA

\begin{abstract}
Many ultra-high-energy neutrino-detection experiments seek radio wave signals from neutrino interactions deep within the polar ice, and an understanding of in-ice radio wave propagation is therefore of critical importance. The parabolic equation (PE) method for modeling the propagation of radio waves is a suitable intermediate between ray tracing and finite-difference time domain (FDTD) methods in terms of accuracy and computation time. The RET collaboration has developed the first modification of the PE method for use in modeling in-ice radio wave propagation for ultra high energy cosmic ray and neutrino detection experiments. In this proceeding we will detail the motivation for the development of this technique, the process by which it was modified for in-ice use, and showcase the accuracy of its results by comparing to FDTD and ray tracing.
\end{abstract}

$37^{\text {th }}$ International Cosmic Ray Conference (ICRC 2021)

July 12th-23rd, 2021

Online - Berlin, Germany

\footnotetext{
${ }^{*}$ Presenter
} 


\section{Introduction}

Many current and next-generation experiments for ultra-high-energy (UHE) neutrino detection employ radio based methods to sparsely instrument large detection volumes [1-6]. Several experimental efforts use the Antarctic or Arctic ice as their detection medium, either directly instrumenting the ice or monitoring the ice from above. Understanding radio wave propagation within the ice is therefore crucial to detector construction and event reconstruction.

To that end, a variety of simulations have been developed to model such propagation [7-10]. Ground penetrating radar [11] and ice cores [12,13] provide a wealth of data on the density of the ice (which is directly translatable to an index of refraction profile [14]) which largely dictates propagation. Simulations relying on geometric optics often use functional approximations to the density profile in order to find fast solutions for propagation. These simulations are often referred to as ray tracing (RT) methods. Such methods are very useful in Monte-Carlo applications where a large number of events must be simulated efficiently. Finite-difference time-domain (FDTD) methods [15] solve Maxwell's equations on a grid, which allows for solutions to radio wave propagation to arbitrary precision. This precision comes at a cost, however, making exact simulations prohibitive for the majority of scenarios of interest to high energy neutrino experiments, where large volumes and long baselines are common (e.g. for a cylindrical volume of $\mathrm{R}$ by $\mathrm{Z}$ with resolution $\mathrm{d}$ memory scales with $\mathrm{RZd}^{2}$ and computational time scales with $\mathrm{RZd} \mathrm{d}^{3}$ ). A third method is presented here, the parabolic equation (PE) approximation method $[16,17]$, that is faster than FDTD (at the expense of some accuracy), and slower than RT, but with the ability to capture effects (see for example Figure 1) that RT methods do not.

In this proceeding we briefly summarize the PE method, its adaptation to in-ice use, and some example comparison to other methods.

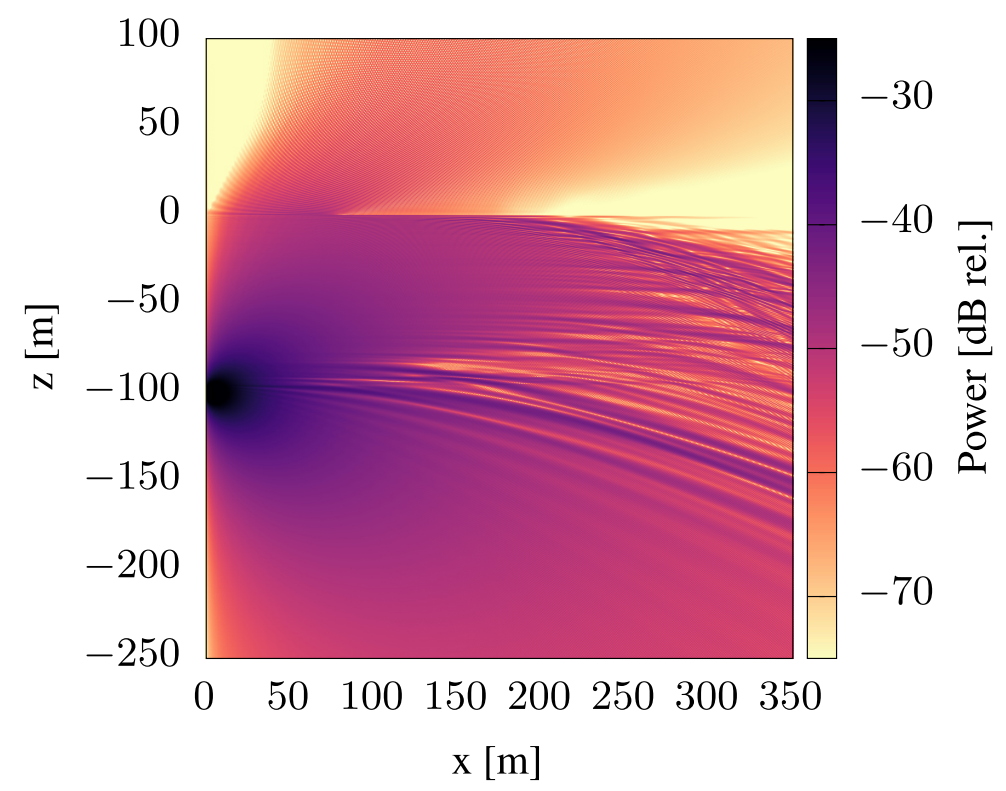

Figure 1: An example output from the parabolic equation solver for a a data-driven index of refraction profile, as explained in the text. The PE solver shows detailed structure in the field arising as a result of small density fluctiations in the index of refration profile. 


\section{Parabolic Equation Methods}

The parabolic approximation is, simply, an approximate solution to the wave equation that solve for the fields propagating in only one of two directions (the paraxial direction). When this approximation is made, the form of the solution is an iterative one, meaning that the solution at some range (horizontal displacement) step is only dependent upon the solution at a previous range step, thus eliminating the need to solve the entire domain at every step, as needed in exact solutions. This greatly increases computational speed.

However, it also introduces errors. The key to the PE method is to find an approximation that minimizes errors. These methods have existed for many decades, and have been applied to in-air radio wave propagation [18] and in-water acoustic propagation [16, 19, 20].

The in-ice approximation is first presented in Ref. [17], and has been incorporated into a simple code, [21]. The derivation of the parabolic approximation and the in-ice form of the expressions are shown in full detail in the appendix of Ref. [17].

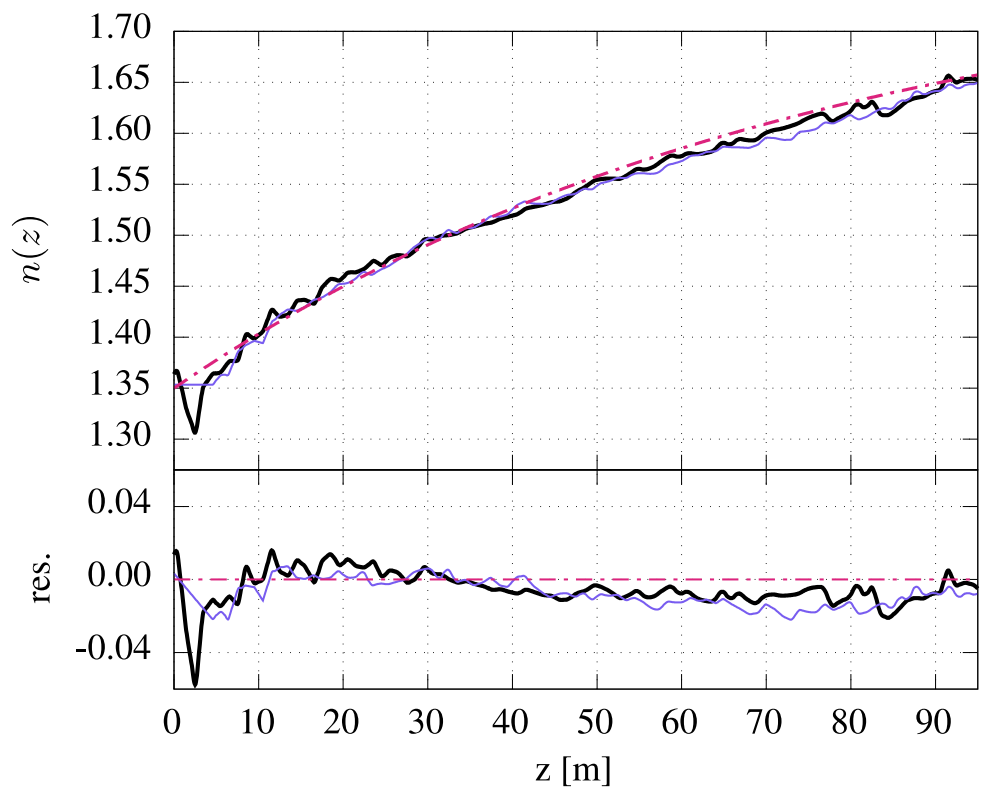

Figure 2: The index of refraction as a function of the depth coordinate $z$. Shown are 2 different measurements from the SPICE core at the south pole, as well as a typical functional profile used in RT codes. residuals below.

\section{Ice profile}

To outline the need for PE simulations, here we briefly discuss the profile of polar ice. Polar ice sheets are compacted precipitation. Each annual layer sits on top of those below, compacting them over the years. This results in a density gradient that is less dense at the surface, and solid ice deep below. The transition region is called the firn, where the precipitation is being slowly compacted.

This profile is generally described by an exponential function, that reaches an asymptotic value equal to that of ice at the bottom of the firn. However, the individual annual layers are not all the 
same thickness, nor do they compact evenly. There are, therefore, fluctuations from this functional profile that will affect radio propagation. The smoothed density $\rho$ provides an index of refraction profile as a function of the depth coordinate $z$ via the expression: $n(z)=1+0.845 \rho(z)$. The PE method attempts to account for realistic fluctuations on top of the smoothed density profile while still being relatively computationally efficient.

Figure 2 shows two different index of refraction profiles derived from SPICE core data [12] at the south pole, overlaid on top of a typical functional profile used in RT codes. The question of note is: how much do these fluctuations affect radio propagation, and how do we simulate it?

\section{Results}

We show in this section several figures comparing different simulation methods. For figures in this section, please see figure captions for the geometry of the setup. The first comparison, Fig. 3, shows a comparison between FDTD, PE, and RT for the case where the ice profile is functional (i.e. without any density fluctuations).

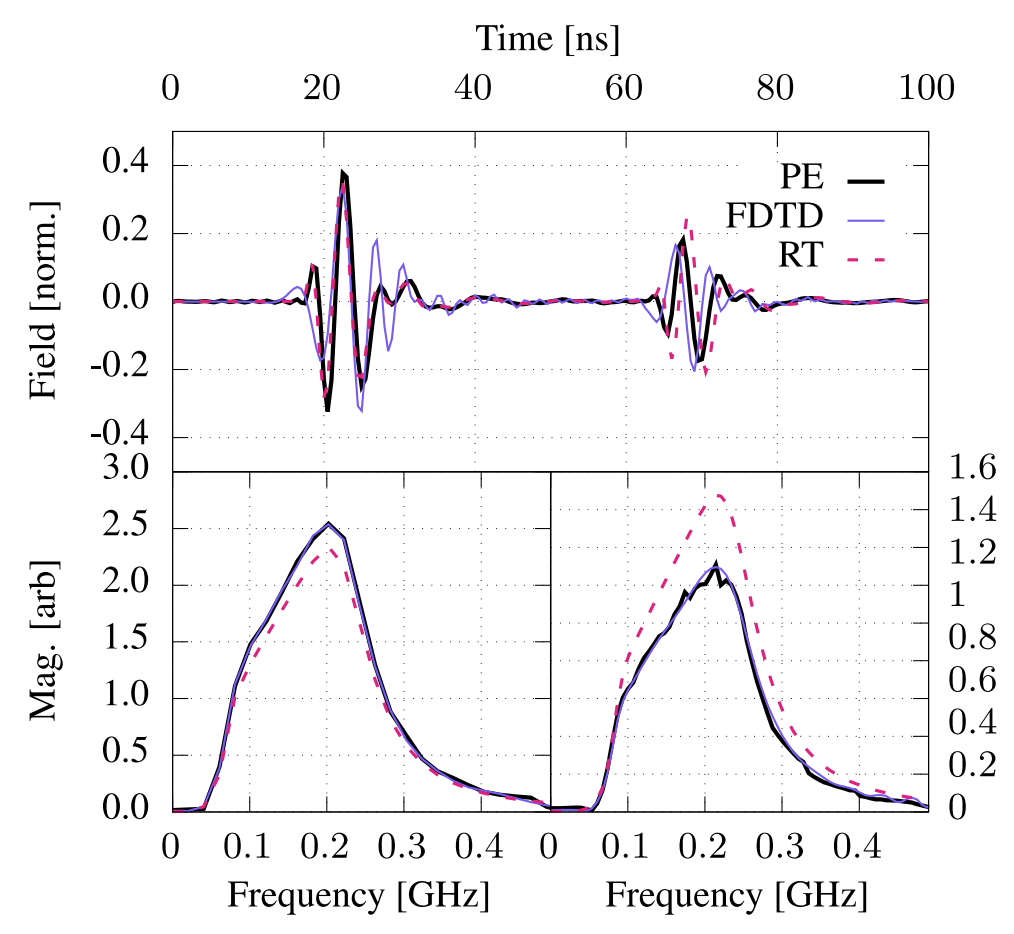

Figure 3: A comparison between FDTD, RT, and PE methods for a functional index of refraction profile. The transmitter is at -30 meters depth and 0 range (horizontal displacement), and the receiver is at -25 meters depth and 100 meters in range.

Fig. 4 shows the same transmitter and receiver geometry as Fig. 3, but with a density profile that is derived from SPICE core measurements. Notice how the field simulations (FDTD and PE) show qualitative agreement, and deviate from the RT methods, particularly in the frequency domain. This deviation is expected, as the density fluctuations will introduce frequency-specific effects, owing to their varying length scales. 


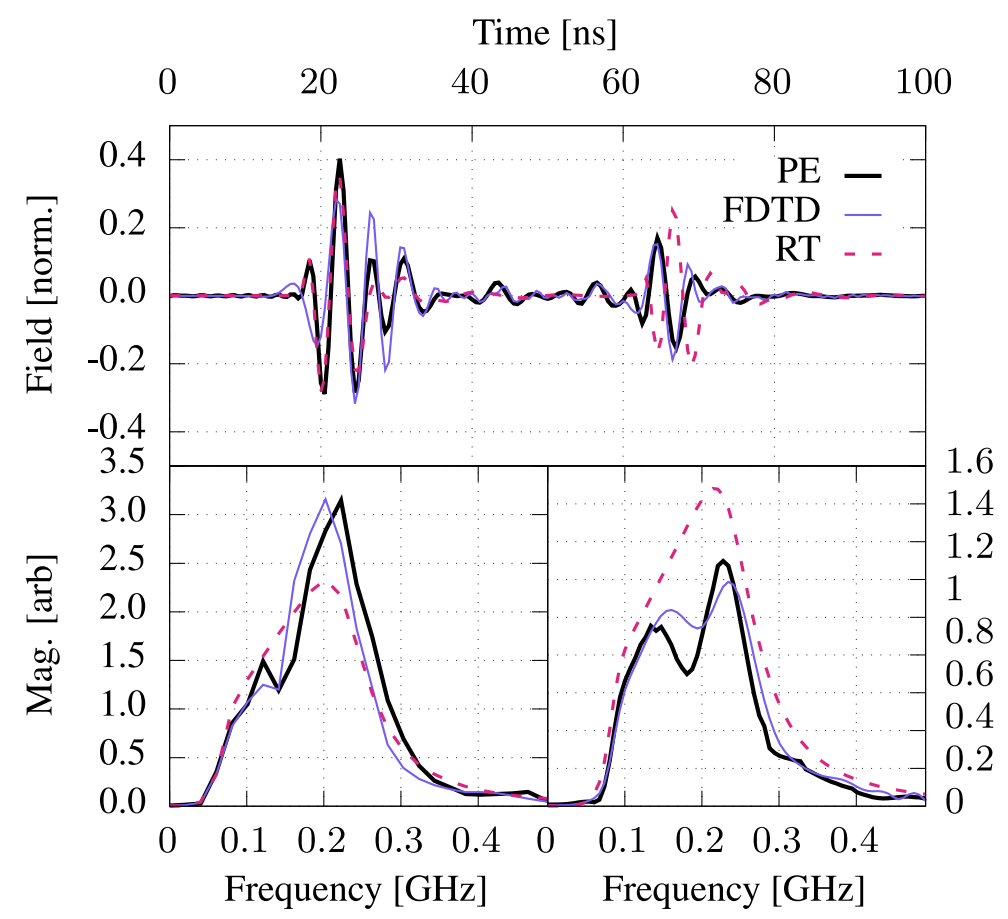

Figure 4: A comparison between FDTD, RT, and PE methods for a data driven index of refraction profile from SPICE core data. The transmitter is at -30 meters depth and 0 range, and the receiver is at -25 meters depth and 100 meters in range.

Fig. 5 shows a map of the spectrum for a receiver as it is lowered through the ice. The transmitter is $1 \mathrm{~km}$ away and $1 \mathrm{~km}$ deep. The evident fluctuations indicate that the received signal spectrum depends on the position of the receiver, which is of general interest to all in-ice radio based neutrino detectors.

\section{Implications for the Radar Echo Telescope for Cosmic Rays}

The Radar Echo Telescope for Cosmic Rays (RET-CR) [22] is a forthcoming experiment to test the radar echo method for neutrino detection (for details, please see Ref. [22] and our other contributions to this conference). Because this experiment will be located in the firn, we need to have a deep understanding of radio wave propagation, which begins with the work presented here. In-situ density studies, coupled with a multi-pronged simulation approach including PE methods, will allow us to understand the propagation at our eventual site, and optimize detector geometry.

\section{Conclusions}

We have presented the first effort to adapt parabolic equation methods to in-ice radio wave propagation. We have shown simulation comparisons to other existing simulation methods, demonstrating the PE method's utility. We have discussed implications for current and future experiments. We conclude that the PE method is useful for simulating radio wave propagation in the ice. 


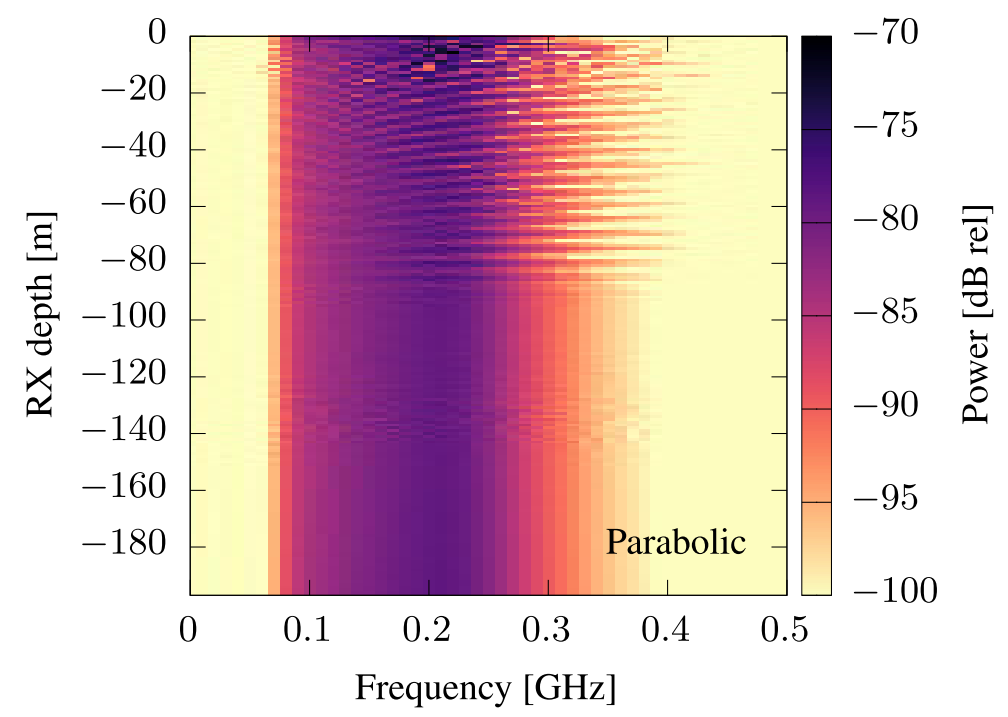

Figure 5: The received spectrum as a function of receiver depth for a transmitter at a depth of $1 \mathrm{~km}$ and a range of $1 \mathrm{~km}$.

\section{References}

[1] P. Allison et al., Design and Initial Performance of the Askaryan Radio Array Prototype EeV Neutrino Detector at the South Pole, Astropart. Phys. 35 (2012) 457 [1105 . 2854].

[2] S.R. Klein et al., A radio detector array for cosmic neutrinos on the ross ice shelf, IEEE Transactions on Nuclear Science 60 (2013) 637.

[3] ANITA collaboration, The Antarctic Impulsive Transient Antenna Ultra-high Energy Neutrino Detector Design, Performance, and Sensitivity for 2006-2007 Balloon Flight, Astropart. Phys. 32 (2009) 10 [0812 . 1920].

[4] S. Wissel et al., Prospects for high-elevation radio detection of $>100 \mathrm{PeV}$ tau neutrinos, JCAP 11 (2020) 065 [2004.12718].

[5] J.A. Aguilar et al., The Next-Generation Radio Neutrino Observatory-Multi-Messenger Neutrino Astrophysics at Extreme Energies, 1907.12526.

[6] Q. Abarr et al., The Payload for Ultrahigh Energy Observations (PUEO): A White Paper, 2010.02892.

[7] ARA-collaborationhttps://github.com/ara-software/AraSim (2020) .

[8] C. Glaser et al., NuRadioMC: Simulating the radio emission of neutrinos from interaction to detector, Eur. Phys. J. C 80 (2020) 77 [1906.01670].

[9] C. Glaser, A. Nelles, I. Plaisier, C. Welling, S.W. Barwick, D. García-Fernández et al., NuRadioReco: A reconstruction framework for radio neutrino detectors, Eur. Phys. J. C 79 (2019) 464 [1903.07023]. 
[10] C. Deaconu, A.G. Vieregg, S.A. Wissel, J. Bowen, S. Chipman, A. Gupta et al., Measurements and Modeling of Near-Surface Radio Propagation in Glacial Ice and Implications for Neutrino Experiments, Phys. Rev. D98 (2018) 043010 [1805 . 12576].

[11] R.J. Arthern, H.F. J. Corr, F. Gillet-Chaulet, R.L. Hawley and E.M. Morris, Inversion for the density-depth profile of polar firn using a stepped-frequency radar, Journal of Geophysical Research: Earth Surface 118 (2013) 1257

[https://agupubs.onlinelibrary.wiley.com/doi/pdf/10.1002/jgrf.20089].

[12] D.A. Winski, T.J. Fudge, D.G. Ferris, E.C. Osterberg, J.M. Fegyveresi, J. Cole-Dai et al., The sp19 chronology for the south pole ice core-part 1: volcanic matching and annual layer counting, Climate of the Past 15 (2019) .

[13] K.A. Casey, T. Fudge, T. Neumann, E. Steig, M. Cavitte and D. Blankenship, The $1500 \mathrm{~m}$ south pole ice core: recovering a 40 ka environmental record, Annals of Glaciology 55 (2014) 137.

[14] G.d.Q. Robin, Velocity of radio waves in ice by means of a bore-hole interferometric technique, Journal of Glaciology 15 (1975) 151.

[15] A. Taflove and S.C. Hagness, Computational electrodynamics: the finite-difference time-domain method, Artech house (2005).

[16] F.D. Tappert, The parabolic approximation method, in Wave propagation and underwater acoustics, pp. 224-287, Springer (1977).

[17] RADAR ECHO Telescope collaboration, Modeling in-ice radio propagation with parabolic equation methods, 2011.05997.

[18] G. Apaydin and L. Sevgi, The split-step-fourier and finite-element-based parabolic-equation propagation-prediction tools: Canonical tests, systematic comparisons, and calibration, IEEE Antennas and Propagation Magazine 52 (2010) 66.

[19] M. Feit and J. Fleck, Light propagation in graded-index optical fibers, Applied optics 17 (1978) 3990.

[20] J.A. Fleck, J. Morris and M. Feit, Time-dependent propagation of high energy laser beams through the atmosphere, Applied physics 10 (1976) 129.

[21] S. Prohirahttps://github.com/prchyr/paraPropPython (2020) .

[22] S. Prohira et al., The Radar Echo Telescope for Cosmic Rays: Pathfinder Experiment for a Next-Generation Neutrino Observatory, 2104.00459. 


\section{Full Authors List: Radar Echo Telescope Collaboration}

S. Prohira ${ }^{1}$, K.D. de Vries ${ }^{2}$, P. Allison ${ }^{1}$, J. Beatty ${ }^{1}$, D. Besson ${ }^{3,4}$, A. Connolly ${ }^{1}$, P. Dasgupta ${ }^{5}$, C. Deaconu ${ }^{6}$, S. De Kockere ${ }^{2}$,

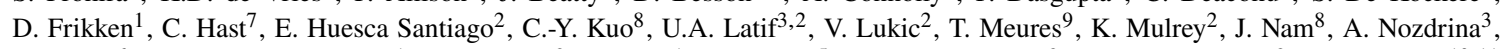
E. Oberla ${ }^{6}$, J.P. Ralston C. Sbrocco ${ }^{1}$, R.S. Stanley ${ }^{2}$, J. Torres ${ }^{1}$, S. Toscano ${ }^{5}$, D. Van den Broeck ${ }^{2}$, N. van Eijndhoven ${ }^{2}$, and S. Wissel ${ }^{10,11}$

${ }^{1}$ Department of Physics, Center for Cosmology and AstroParticle Physics (CCAPP), The Ohio State University, Columbus OH, USA

${ }^{2}$ Vrije Universiteit Brussel, Brussel, Belgium

${ }^{3}$ University of Kansas, Lawrence, KS, USA

${ }^{4}$ National Research Nuclear University, Moscow Engineering Physics Institute, Moscow, Russia

${ }^{5}$ Université Libre de Bruxelles, Brussels, Belgium

${ }^{6}$ Enrico Fermi Institute, Kavli Institute for Cosmological Physics,Department of Physics, University of Chicago, Chicago, IL, USA

${ }^{7}$ SLAC National Accelerator Laboratory, Menlo Park, CA, USA

${ }^{8}$ National Taiwan University, Taipei, Taiwan

${ }^{9}$ University of Wisconsin-Madison, Madison, WI, USA

${ }^{10}$ Departments of Physics and Astronomy \& Astrophysics,Institute for Gravitation and the Cosmos, Pennsylvania State University, University Park, PA, USA

${ }^{11}$ California Polytechnic State University, San Luis Obispo CA, USA 